ный агроинженерный иеетр ВИМ, Россия.

109428, г. Москва, 1-й Институтский пр-д, 5.

Тел.: (915) 319-21-69.

Коцарь Юрий Алексеевич, $\partial$ - $р$ тех. наук, проф. кафедры «Техносферная безопасность и транспортно-технологические машины», Саратовский государственный аграрный университет имени Н.И. Вавилова. Россия.
410056, г. Саратов, ул. Советская, 60.

Тел.: (8452) 74-96-63.

Ключевые слова: зерно; зерноочистительная машина; автоматизация технологического процесса; система управления; алгоритм работы.

\title{
AUTOMATED PROCESS CONTROL SYSTEM FOR A GRAIN CLEANING MACHINE
}

Zagoruiko Mikhail Gennadyevich, Candidate of Technical Sciences, Senior Researcher, Federal Scientific Agroengineering Center VIM. Russia.

Starostin Ivan Aleksandrovich, Candidate of Technical Sciences, Senior Researcher, Federal Scientific Agroengineering Center VIM. Russia.

Kotsar Yuriy Alekseevich, Doctor of Technical Sciences, Professor of the chair "Technosphere Safety and Transport and Technological Machinery", Saratov State Agrarian University named after N.I. Vavilov. Russia.

Keywords: grain; grain cleaning machine; process automation; control system; algorithm of operation.

The article discusses the main problems that arise when working with commercially available grain cleaning ma- chines "Petkus-Giant" K531. To solve these problems, we offer a set of removable equipment that allows automating the grain cleaning process with minimal financial costs, and a control system that monitors the content of weed impurities in the cleaned grain, commercial grain in the exits of light impurities from the first and second aspiration channels, the exit of small impurities (the passage of the lower sieve) and the exit of large impurities (the descent from the upper sieve). The proposed set includes a level sensor, cameras, a processor with a control unit, and actuators in the form of servos. The algorithm of operation of the process control system of the grain cleaning machine is described. Continuous monitoring and adjustment of the parameters of the process flow by the control system allows one to meet all the requirements.

\section{ОЦЕНКА ВЕЛИЧИНЫ СВЧ-МОЩНОСТИ ГРУППИРОВАТЕЛЯ В ПОВТОРНОМ МАГНИТНОМ ПОЛЕ}

\author{
КУЗЬМИН Константин Анатольевич, Московский государственный университет технологий \\ и управления имени К.Г. Разумовского (Первый казачий университет) \\ моРОзов Сергей Михайлович, Московский государственный университет технологий \\ и управления имени К.Г. Разумовского (Первый казачий университет)
}

ПАВЛОВ ИГОРЬ ВАЛЕНТИНОВИЧ, Московский государственный университет технологий и управления имени К.Г. Разумовского (Первый казачий университет)

РЕУТ ВЛАДИМИР АНТОНОВИЧ, Московский государственный университет технологий и управления имени К.Г. Разумовского (Первый казачий университет)

БАЛМАШНОВА ЕЛЕНА ВИТАЛЬЕВНА, Национальный исследовательский Московский государственный строительный университет

\section{МОРОЗОВ МИХАИЛ СЕРГЕЕВИЧ, Московский авиацһинный институт}

В статъе рассматривается клистронный метод группировки в повторном магнитном поле. В длинноволновом диапазоне, кроме гирокона и магникона, исполъзуются СВЧ-тетроды и многорезонаторные клистроны. Обычные клистроны в дециметровом диапазоне имеют значительные размеры, и их применение становится проблематичным, а СВЧ-тетроды имеют значительный уровенъ мощности, но невысокий кПд и коэффициент усиления.

Введение Первый этап развития релятивистской электроники приходится на 1950-60-е гг., когда теоретически и экспериментально изучались различные способы генерации колебаний в неосвоенных тогда миллиметровом и субмиллиметровом диапазонах длин волн с помощью ускорителей-группирователей, создающих сгустки электронов, и излучателей различного типа. В качестве ускорителей-группирователей применялись линейные ускорители, ребатроны и микротроны. Излучение сгустков наблюдалось в магнитном ондуляторе и гармонотроне, в черенковском излучателе и генераторе переходного излучения, в открытых резонаторах и специальных волноводах. Были получены когерентные колебания миллиметрового и субмиллиметрового диапазонов с мощностью излучения до нескольких ватт $[4,7]$.

Исследования индуцированного излучения свободных электронов послужили основой приборов на классическом индуцированном (излучении свободных электронов) - электронных 
мазеров [1-3]. Наибольшее распространение получил слабо релятивистский МЦР, особенно одна из его высокоэффективных разновидностей - гиротрон. Гиротрон содержит адиабатическую термоэмиссионную магнетронную пушку и слабонерегулярный волновод. В гиротроне происходит взаимодействие потока возбужденных слаборелятивистских электронных осцилляторов с одной из собственных волн волновода вблизи частоты отсечки. Развитие гиротронов Горьковской-Нижегородской школы академика А.И.Гапонова привело к созданию источников миллиметрового излучения с мощностью на 2-3 порядка превосходящей другие приборы, работающие в коротковолновой части СВЧдиапазона.

Методика исследований. Альтернативой клистронным методам группировки может служить метод группировки в повторном магнитном поле (ПМП). В его основе лежит известный эффект сильной зависимости радиуса поворота релятивистского электрона от его относительной энергии $\gamma$ при движении в поперечном (поворотном) магнитном поле с индукцией $B$ :

$$
\mathrm{v}_{0}=\frac{m_{0} c}{e B} \sqrt{\gamma_{0}^{2}-1}
$$

Возможность создания группирователя на основе этого эффекта первоначально рассмотрена в ряде работ зарубежных авторов. Идея предложенного метода заключается в том, что промодулированные по энергии электроны, попадая в поворотное магнитное поле, движутся по окружностям различного радиуса, пропорционально $\gamma$, т.е. проходят различные пути практически с одинаковой скоростью. Благодаря этому расстояния между первоначально равномерного отстоящими друг от друга электронами сокращаются после полного поворота в магнитном поле, т.е. происходит группировка. Схема такого группирователя показана на рис. 1 , а. РЭП модулируется по энергии в модуляторе резонатора 1 , затем группируется в поворотном кольце 2 . На выходе из поворотного кольца электронный поток представляет собой последовательность сгустков, от которых в дальнейшем осуществляется отбор энергии выходной системой 3.

Результаты исследований. Наряду с группирователем, реализующим способ клистронного группирования при непрерывном ускорении, для релятивистского клистрона рассматривался группирователь РЭП в поворотном магнитном поле. Принцип группирования релятивистского электронного потока в ПМП основан, как отмечалось, на том, что радиус дружности, по которой движется заряженная частица в постоянном

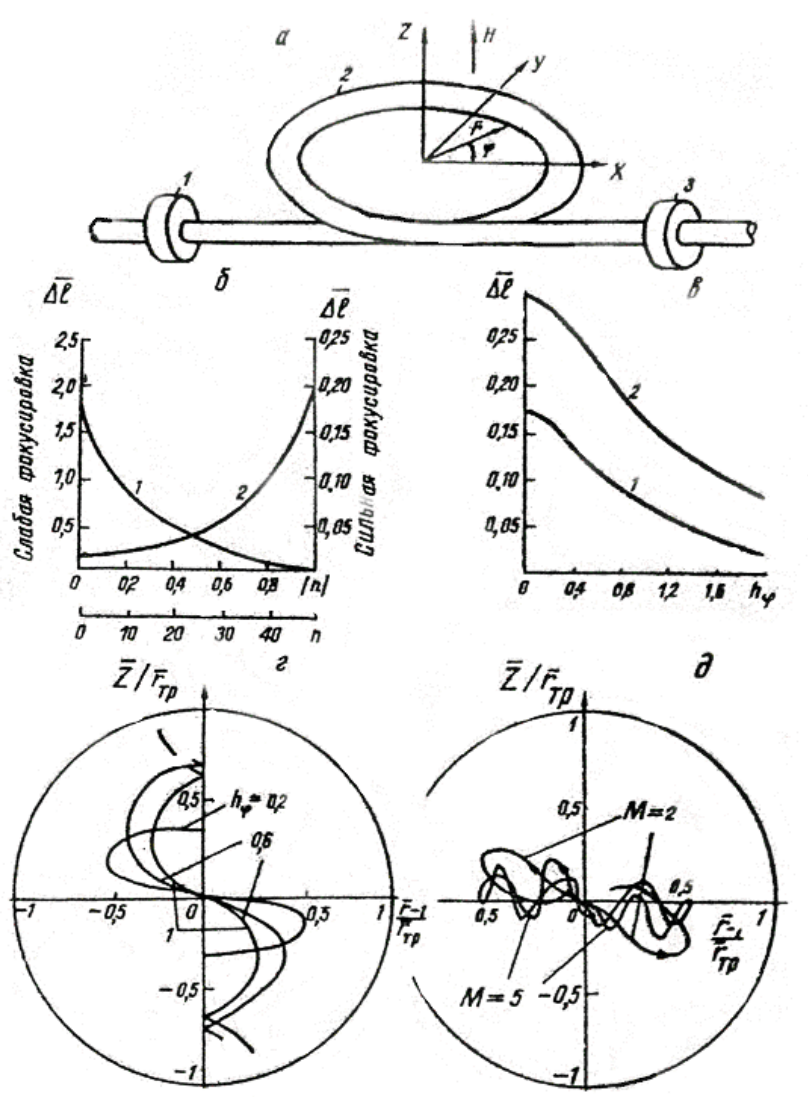

Рис. 1. Схема поворотного группирователя и зависимости, характеризующие движение электронов в нем

магнитном поле с силовыми линиями, перпендикулярными этой окружности, зависит от массы частицы, а в релятивистском случае - от ее энергии. На поворотное магнитное поле наложено периодическое фокусирующее.

Получив на входе в группирователе модуляцию по энергии, частицы пучка начинают двигаться по окружностям разного радиуса проходя, таким образом, за одно и то же время разные пути.

Это приводит к образованию электронных сгустков. Плотность сгустков по мере их движения в петлевом группирователе увеличивается, одновременно с этим растут поля кулоновского расталкивания.

Оценим величину активной мощности, которая должна поступать в модулирующий резонатор. Ускорения и торможения электронов переменным полем ведет при конечных углах пролета в зазоре не только к появлению скоростной модуляции, но и к процессу группирования. Из-за большего времени пролета заторможенных электронов через зазор они замедляются меньше, чем увеличивают свою скорость быстрые электроны, пролетающие зазор за меньшее время. Переменное поле резонатора в среднем за период теряет энергию на взаимодействие с электронами, которая и восполняется поступающей от генератора энергией $[5,6]$. 
Известно, что для получения максимального значения передаваемой мощности от генератора во входной резонатор группирователя связь между ними подбирается так, чтобы вносимое в резонатор сопротивление было равно собственному сопротивлению резонатора при резонансе. В результате нагрузочное сопротивление резонатора падает до $R_{\mathrm{H}} / 2$ и вводимая в него мощность

$$
P_{\mathrm{x}}=\frac{1}{2} \frac{U_{m}^{2}}{\frac{1}{2} R_{\mathrm{H}}}=\frac{U_{m}^{2}}{R_{\mathrm{H}}},
$$

где $U_{m}$ - напряжение на зазоре;

$$
\begin{gathered}
R_{\mathrm{H}}=\frac{R_{e} R_{\mathrm{o \jmath}}}{R_{e}+R_{\mathrm{o \jmath}}}, \\
R_{e}=\frac{1}{G_{e}},
\end{gathered}
$$

где $G_{e}$ - активная проводимость РЭП;

$R_{\text {оэ }}^{e}$ эквивалентное сопротивление резонатора при резонансе.

Таким образом, (2) можно представить в виде

$$
P_{\text {вх }}=P_{\text {вх.собств }}+P_{\text {вх.эл }},
$$

где $P_{\text {вх.собств }}$ - мощность, переходящая в тепловые потери резонатора,

$$
P_{\text {вх.собств }}=\frac{1}{2} \frac{U_{m}^{2}}{R_{\text {эо }}} ;
$$

$P_{\text {вх.эл }}-$ мощность, переходящая в кинетическую энергию потока при его модуляции по скорости;

$$
P_{\text {в.эл }}=\frac{1}{2} U_{m}^{2} G_{e} .
$$

$G_{e}$ определяется в данном случае соотношением:

$$
G_{e}=\frac{I_{0}}{U_{0}} K_{m} \psi\left(\xi_{d c}\right)
$$

где $I_{0}$ - постоянная составляющая точка пучка; $U_{0}$ - энергия пучка, эВ; $\psi$ - функция, зависящая от угла пролета $\xi_{d c} ; K_{m}-$ множитель, учитывающий релятивистский эффект,

$$
\begin{gathered}
\kappa_{m}=\frac{2}{\gamma_{0}\left(1+\gamma_{0}\right)} . \\
\gamma_{0}=\frac{1}{\sqrt{1-\frac{v^{2}}{c^{2}}}}=1+1,965 \cdot 10^{-6} U_{0} .
\end{gathered}
$$

Максимальное значение составляет 0,2 при $\xi_{d c}=\pi$, когда в качестве этой функции берется $\psi_{1}$ или $\psi_{4}$.

Таким образом, выражение для $P_{\text {вх.эл }}$ примет вид

$$
P_{\text {вх.эл }}=\frac{I_{0} U_{m}^{2}}{U_{0}} \frac{2}{\gamma_{0}\left(1+\gamma_{0}\right)} \psi \text {. }
$$

Приняв $\psi=0,2$ из (2), получим:

$$
P_{\text {вх.эл }}=\frac{I_{0}}{U_{0}} \cdot 0,4\left(\frac{0,04 \cdot 10^{6}}{1,965}\right)\left(\frac{\gamma_{0}}{\gamma_{0}+1}\right) .
$$

\begin{tabular}{|c|c|c|c|c|c|c|}
\hline$I_{0} / E_{0}$ & $1 \mathrm{MэB}$ & $2 \mathrm{MэB}$ & 3 МэВ & 5 МэВ & 6 МэВ & $8 \mathrm{Mэ}$ \\
\hline $50 \mathrm{~A}$ & 6 кВт & 3,3 кВт & 2,4 кВт & $1,5 \kappa B \mathrm{~T}$ & 1,3 кВт & 1 кВт \\
\hline $100 \mathrm{~A}$ & 12 кВт & 6,6 кВт & 4,8 кВт & 3 кВт & 2,6 кВт & 2 кВт \\
\hline $150 \mathrm{~A}$ & 18,6 кВт & 10,3 кВт & 7,2 кВт & $4,5 \kappa B \mathrm{~T}$ & 3,8 кВт & 3 кВт \\
\hline $180 \mathrm{~A}$ & 22,3 кВт & 12,4 кВт & 8,7 кВт & 5,4 кВт & 4,6 кВт & 3,5 кВт \\
\hline $200 \mathrm{~A}$ & 24,8 кВт & 13,8 кВт & 9,6 кВт & 6 кВт & 5,2 кВ т & $3,9 \kappa \mathrm{BT}$ \\
\hline
\end{tabular}

Или с учетом (3):

$$
P_{\text {вх.эл }}=\frac{I_{0}}{U_{0}} \cdot 0,4\left(\frac{0,04 \cdot 10^{6}}{1,965}\right) \frac{1+1,965 \cdot 10^{-6} U_{0}}{2+1.965 \cdot 10^{-6} U_{0}} .
$$

Результаты расчета $P_{\text {вх.эл}}$, т.е. активной мощности, поступающей в модулирующий резонатор для различных значений тока и энергии пучка, сведены в таблице.

Из полученных результатов видно, что величина необходимой для модуляции пучка мощ-

Результаты расчета активной мощности, поступающей в модулирующий резонатор 
ности при увеличении $U_{0}$ уменьшается.

Это объясняется влиянием релятивистских эффектов. Максимальное значение мощности, обеспечивающей модуляцию в ПМП пучка с энергией $1-8$ МэВ и током $50 \div 200$ А составляет 25 кВт.

Как было отмечено, на входе в группирователь с ПМП должен быть установлен резонатор, согласованный с выходным трактом магнетронного генератора при различных параметрах РЭП. С этой целью были проведены экспериментальные исследования волноводного резонатора. Его конструкция приведена на рис. 2. Резонатор представляет собой отрезок волновода прямоугольного сечения размером $72 \times 20$ мм² $^{2}$ в широкие стенки которого вварены отрезки пролетных труб для подсоединения к электронопроводу ускорителя. Изменение частоты волноводного резонатора осуществляется с помощью гибкой мембраны, перемещаемой с помощью штока сильфонного механизма перестройки. Для согласования волноводного резонатора с трактом генератора использован плавный волноводный трансформатор. Вакуумная полость резонатора отделена от атмосферы слюдяным волноводным окном. Предел перестройки частоты при перемещении плунжера составляет $2890 \div 3080$ МГц, добротность - 450 .

Однако использование такой конструкции модулирующего резонатора представляется нецелесообразным из-за нарушения симметрии электромагнитного поля в нем, что может привести к увеличению амплитуды колебаний электронного пучка и ухудшению его токопрохождения.

Наилучшим образом условиям, обеспечивающим оптимальное группирование РЭП в ПМП, соответствует резонатор системы отбора мощности, используемый в качестве модулирующего. Распределение поля внутри него обладает круговой симметрией относительно оси электронопровода; конструкция резонатора хорошо рассеивает тепло при больших мощностях без пробоев.

Для обеспечения нормальной работы группирователя модулирующего РЭП в ПМП необходимо знать допустимую величину входной мощности, выше которой в модулирующем резонаторе развиваются СВЧ-пробои, шунтирующие электронный пучок.

Начало развития пробоя при увеличении напряженности поля в озонаторе выражается в появлении темнового тока. Ток электронов поверхности резонатора является результатом автоэлектронной эмиссии с больших и острых выступов, значительно превышающих по размерам неровности, получающиеся при механической обработке поверхности. Кроме того, значительная часть темнового тока может быть результа- том автоэлектронной эмиссии с участков поверхностей, имеющих пониженную работу выхода из-за наличия оксидных пленок и загрязнений. Ток автоэлектронной эмиссии резко возрастает с увеличением напряженности поля, прохождение темнового тока сопровождается возникновением рентгеновского излучения, интенсивность которого экспоненциально растет с увеличением напряженности поля. Темновой ток распределен по поверхности резонатора крайне неравномерно и к значительной степени эмитируется с небольших участков поверхности $0,5-1,0$ мм²$^{2}$. На величину темнового тока существенное влияние оказывает частота обработки поверхности, а также чистота материала, из которого он изготовлен. Протекание темнового тока сопровождается голубовато-фиолетовым свечением, интенсивность которого растет с напряженностью поля и силой темпового тока.

Дальнейшее увеличение напряженности электрического поля приводит к искрению. Пробои сопровождаются яркими искрами, а также интенсивными и кратковременными вспышками рентгеновского излучения. Темновой ток и искрение сопровождаются отрывов микрочастиц материала с поверхности резонатора. Материал переносится в виде электрически заряженных частиц размером 3-5 мкм. Интенсивность разрушения поверхности примерно пропорциональна энергии запасенной в резонаторе. На внутренней поверхности резонатора в результате искрения образуются кратеры диаметров до 0,7 мм, от которых вглубь материала идут трещины до 0,2 мм от поверхности кратеров. На такую же глубину от поверхности кратеров распространяется зона рекристаллизации, что свидетельствует об интенсивном локальном нагреве. Образующиеся острые кромки кратеров трещин приводят к снижению.

Задача состоит в том, чтобы разработать эффективную методику, использующую явления, предшествующие СВЧ-пробою, с тем, чтобы не допустить развития описанных выше процессов, которые в конечном итоге приводят в негодность модулирующий резонатор.

В результате многочисленных экспериментов было установлено, что наиболее эффективными факторами индикации СВЧ-пробоя в его начальной стадии являются: уменьшение длительности и резкое обострение заднего фронта огибающей импульса СВЧ-колебаний в резонаторе, рост мощности отраженной волны.

Испытания, проводимые на макетах резонатора, подтвердили правильность выбранного метода. После демонтажа и разработки резонаторов, проводимой на различных стадиях развития пробоя, было обнаружено, что кратер и трещины образуются в том месте, где на начальном этапе, 
когда изменяется форма и длительность импульса, образуется незначительноепотемнение. Таким образом, темновой ток возрастает настолько, что происходит шунтирование высокочастотного зазора и падение амплитуды напряжения, однако таких явлений, как рентгеновское излучение, свечение, увеличение давления в модулирующей системе, еще не наблюдается.

Методика реализована на установке, схема которой приведена на рис. 2. Источником СВЧмощности служит генератор 1-го типа 1РЛ-110, магнетрон которого МИ-29Б генерирует мощность 1 МВт на частоте $f=2,8$ ГГц с длительностью импульсов $\tau=2,7$ мкс и частотой следования $f_{c}=100$ Гц. Развязывающие устройством являлся ферритовый вентиль 2-го типа 9-ВВВ-4 с ослаблением в прямом направлении $A_{1}=1 д Б$ и в обратном направлении $A_{2}=13 д Б$. Далее установлены две фиксированные аттенюаторы 3, прямоугольного сечения $72 \times 34$ мм, на широкие стенки которых по специальной технологии нанесена смесь карбонильного железа и эпоксидной смолы. Между последним аттенюатором, резонатором 4 установлен НО5, к выходам которого присоединяются калориметрические головки измерителей мощности 6-го и 7-го типа M4-46 либо пондеромоторные электродинамические ваттметры. Задающим использован генератор 8-го типа Г5-54, вырабатывающий импульсы с $f_{\text {с }}=100$ Гц, $\tau=2,7$ мкс, $U=10$ В. Для регистрации огибающей импульса СВЧ-колебаний в резонаторе к его коаксиальному выходу на боковой стенке присоединена детекторная секция 9, соединенная с осциллографом 10.

При подъеме питающего напряжения на выходе генератора 1РЛ-110 получим медленный рост выходной мощности. Начало развития СВЧ-пробоя в резонаторе сопровождается уменьшением длительности и резким обострением заднего фронта импульса, регистрируемого
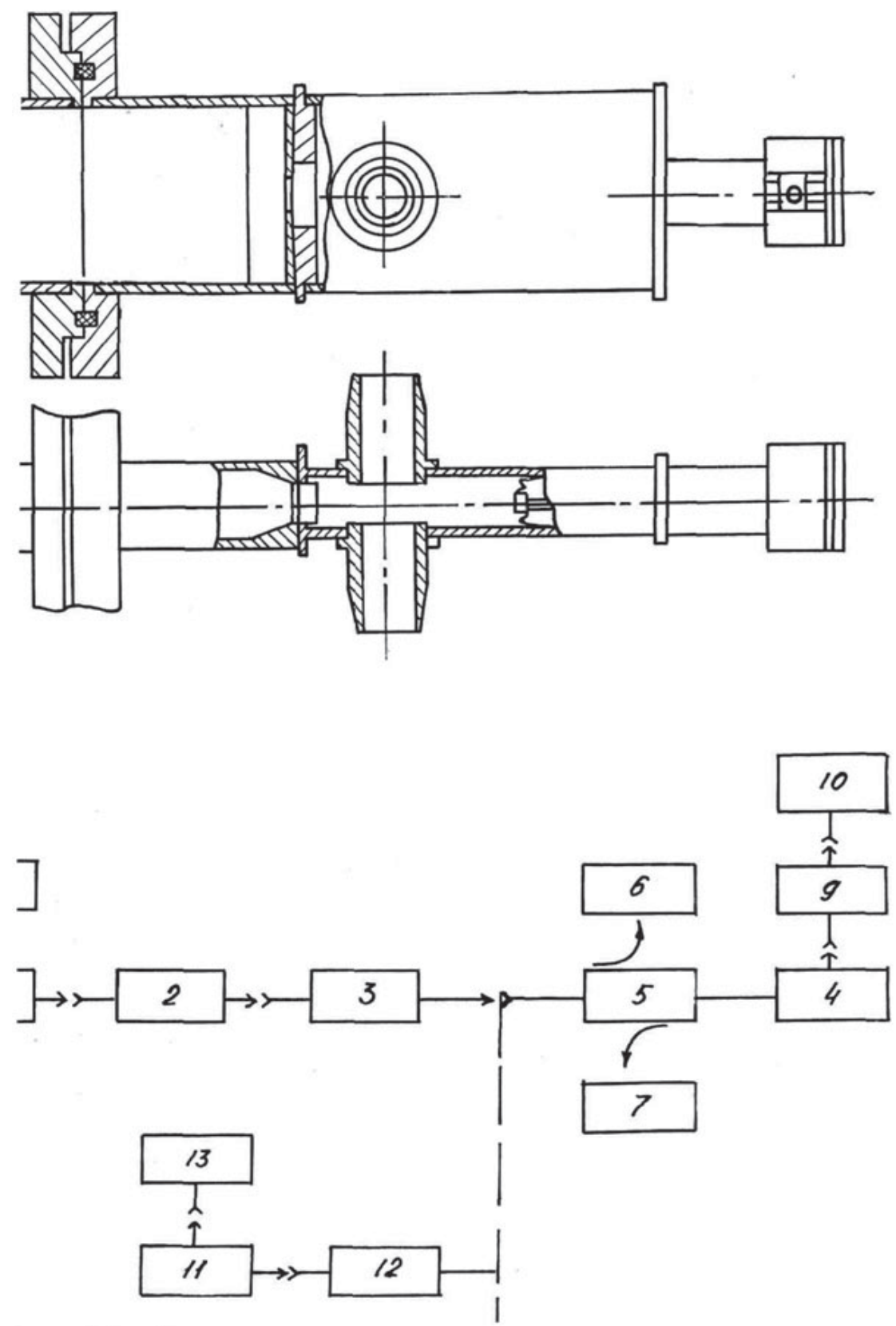

Рис. 2. Резонатор группирователя в ПМПи структурная схема исследований 

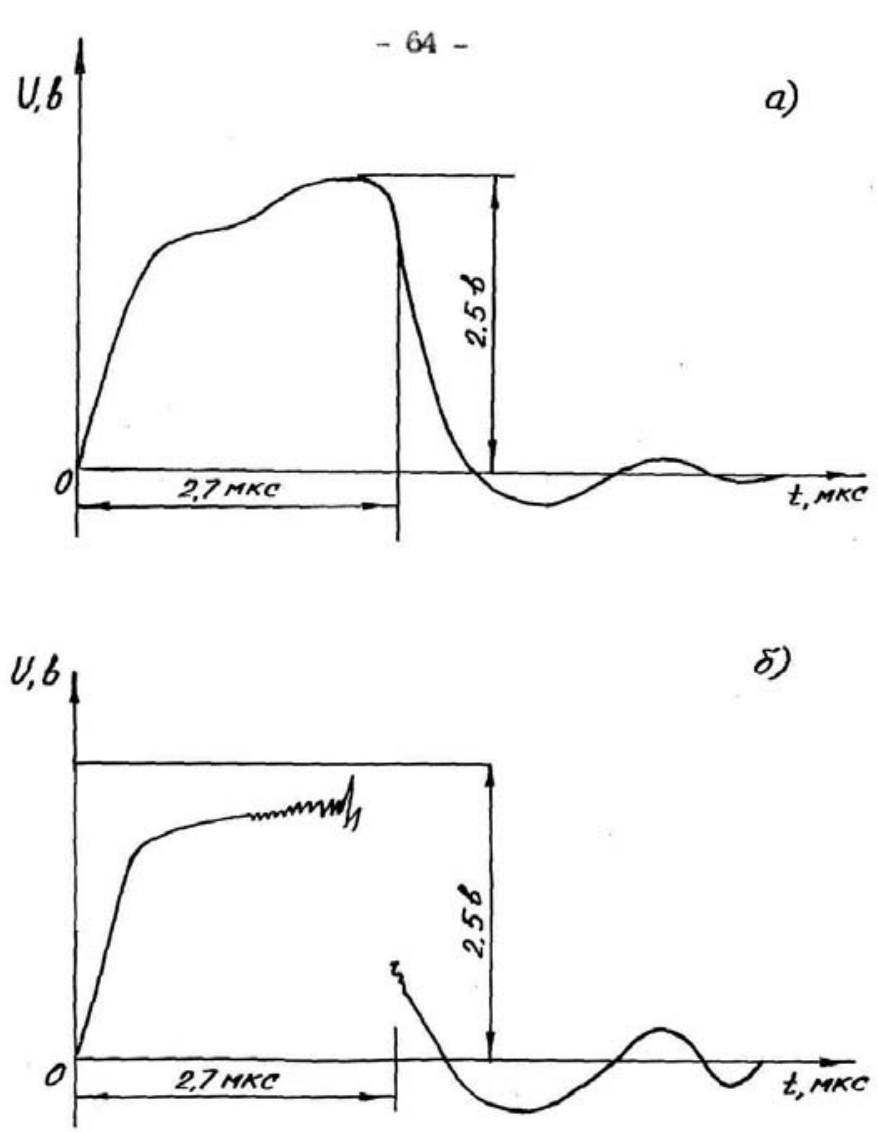

Рис. 3. Осциллограммы огибающей импульса СВЧ-колебаний в резонаторе при нормальной работе (а) и в начале развития пробоя (б)
2. Морозов С., Кузьмин К., Макаров $Г$. Нейросетевой принцип реализации цифровых фильтров // веб-конференция МАТЕС. - 2018. - Вып. 193. C. $102-115$.

3. Расчет параметров и характеристик СВЧ-установки для сушки и обжига кирпича / С.М. Морозов [и др.] // Бюллетень строительной техники. 2018. - № 9. -С. 37-49.

4. Федулов В.И., Морозов С.М. Потери кинетической энергии частицы в неоднородном потенциальном поле // Современное телевидение и радиоэлектроника 19-я Междунар. науч.-техн. конф. - 2011. - С. 299-301.

5. Fedulov V.I. ARW 977788 Emerging Applications of Vacuum-Arc-Produced Plasma, Ion and Electron Beams / edited by E. M. Oks and I. G. Brown (Kluwer Academic Publishers, Dordrecht, the Netherlands) 2003; 213-225.

6. Kroemer H. Electrical and Computer Engineering Department, University of California, Santa Barbara, 2001.

7. Landau L., Lifchic E. The theory of a field. Moscow, 1988. Vol. 2, p. 78-79.

Кузьмин Константин Анатольевич, канд. пед. наук, доцент кафедры «Естественно-научные технические дисциплины и информационные технологии», Московский государственный университет технологий и управления имени К.Г. Разумовского (Первый казана осциллографе (рис. 3), и ростом измеряемой мощности в канале «отраженный» НО. При увеличении $P_{\text {вых }}$ генератора развитие СВЧ-пробоя усиливается, о чем свидетельствуют дальнейшее уменьшение длительности импульса и возрастание отраженной мощности.

Величина допустимой, закачиваемой в модулирующий резонатор мощности регистрируется в канале «падающей» НО; она максимальна, если с ростом величины питающего напряжения генератора ее рост прекращается.

Заключение. Необходимо отметить, что теоретический анализ и методы математического моделирования с использованием ЭВМ позволяют осуществить лишь предварительный расчет таких устройств, в то время как условия разработки диктуют необходимость определения параметров для реальных условий, к тому же ряд характеристик устройств не может быть определен путем моделирования ввиду их сложности. В этих условиях наибольшую практическую ценность приобретают экспериментальные исследования.

\section{СПИСОК ЛИТЕРАТУРЫ}

1. Кузьмин К.А., Морозов С.М. Определение оптимальных режимов работы СВЧ-установки для сушки и обжига строительного кирпича // Инженерный Вестник Дона. - 2018. - № 3. - С. 75-83. чий университет). Россия.

Морозов Сергей Михайлович, канд. техн. наук, доцент, зав. кафедрой «Естественно-научные технические дисциплины и информационные технологии», Московский государственный университет технологий и управления имени К.Г. Разумовского (Первый казачий университет). Россия.

Павлов Игорь Валентинович, канд. пед. наук, доцент кафедры «Естественно-научные технические дисииплины и информационные технологии», Московский государственный университет технологий и управления имени К.Г. Разумовского (Первый казачий университет). Россия.

Реут Владимир Антонович, канд. пед. наук, доцент кафедры «Естественно-научные технические дисииплины и информационные технологии», Московский государственный университет технологий и управления имени К.Г. Разумовского (Первый казачий университет). Россия.

215100, Смоленская обл., г. Вязьма, ул. Ленина, 77.

Тел.: (48131)6-18-66

Балмашнова Елена Витальевна, преподаватель кафедры «Технология и организащия строительного производства», Национальный исследовательский Московский государственный строительный университет. Россия.

129337, г. Москва, Ярославское шоссе, 26.

Тел.: +7 (495) 781-80-07.

Морозов Михаил Сергеевич, ведущий инженер, Московский авиационный институт, Россия. 
125993, г. Москва, Волоколамское шоссе, 4. Тел.: (499) 158-29-77.

Ключевые слова: электронный поток; мощность СВЧ; группирователь; повторное магнит- ное поле; клистронный метод; релятивистский электрон; поперечное магнитное поле; электронные сгустки.

\title{
EVALUATION OF THE MICROWAVE POWER OF A BUNCHER IN A REPEATED MAGNETIC FIELD
}

Kuzmin Konstantin Anatolyevich, Candidate of Pedagogical Sciences, Associate Professor of the chair "Science and Technical Disciplines and Information Technology", K.G. Razumovsky Moscow State University of Technologies and Management (the First Cossack University), Russia.

Morozov Sergey Mikhaylovich, Candidate of Technical Sciences, Associate Professor, Head of the chair "Science and Technical Disciplines and Information Technology", K.G. Razumovsky Moscow State University of Technologies and Management (the First Cossack University), Russia.

Pavlov Igor Valentinovich, Candidate of Pedagogical Sciences, Associate Professor of the chair "Science and Technical Disciplines and Information Technology", K.G. Razumovsky Moscow State University of Technologies and Management (the First Cossack University), Russia.

Reut Vladimir Antonovich, Candidate of Pedagogical Sciences, Associate Professor of the chair "Science and Technical Disciplines and Information Technology", K.G. Razumovsky
Moscow State University of Technologies and Management (the First Cossack University), Russia.

Balmashnova Elena Vitalyevna, Teacher of the chair "Technology and Organization of Construction Production", National Research Moscow State University of Civil Engineering. Russia.

Morozov Mikhail Sergeevich, Leading Engineer, Moscow Aviation Institute. Russia.

Keywords: electronic stream; microwave power; buncher; repeated magnetic field; klystron method; relativistic electron; transverse magnetic field; electronic clots.

The article considers the klystron method of grouping in a repeated magnetic field. In the long-wavelength range, in addition to gyrocon and magnicon, microwave tetrodes and multi-cavity klystrons are used. Conventional klystrons in the decimeter range have significant dimensions, and their application becomes problematic, and microwave tetrodes have a significant power level, but low efficiency and gain ratio.

\section{ПРОБЛЕМЫ СОХРАНЕНИЯ СЕНАЖА В РУЛОНЕ, УПАКОВАННОГО В ПЛЕНКУ}

\author{
КучИН Николай Николаевич, Нижегородский государственный инженерно-экономический \\ университет \\ МАНСУРОВ Александр Петрович, ИПТД филиал ГБОУ ВО НГИЭУ \\ жУжин Максим Сергеевич, Нижегородский государственный инженерно-экономический \\ университет
}

ЛОМАЧЕНКО Оксана Александровна, Нижегородский государственный

инженерно-экономический университет

В статье представлен обзор операций технологического процесса заготовки сенажа в рулоне, упакованного в пленку, а также технические средства, с помощъю которых данные операции выполняются. В результате выявлены достоинства и недостатки технологии заготовки сенажа в рулон, упакованного в пленку, и предложены пути их решения.

Введение. Вопрос создания качественной кормовой базы, которая удовлетворяла бы потребности животных в питательных веществах, а также способствовала достижению высокой продуктивности, является актуальным. Важность данной проблемы отражена на высшем уровне - в Указе Президента РФ №350 от 21.07.2016 г. «О мерах по реализации государственной научно-технической политики в интересах развития сельского хозяйства» на период до 2026 года [11], в котором поставлена задача производства высококачественных кормов за счет внедрения современных конкурентоспособных технологий. При традиционных технологиях консервирования сенажа происходят значительные потери питательных веществ. На данный момент времени применяются разные технологии и способы хранения и заготовки сенажа.
Технология заготовки сенажа в рулоны, упакованного в пленку, является одной из конкурентоспособных как в России, так и зарубежном. Суть данной технологии в том, что скошенные травы быстро и равномерно провяливаются, плотно прессуются и надежно упаковываются в герметичную пленку, что позволяет получить качественный корм с минимальными потерями выращенного урожая.

Затраты на корма в структуре себестоимости продуктов животноводства составляют более $50 \%$, поэтому в условиях современной рыночной экономики производство и использование высококачественных кормов обладают приоритетным и стратегическим значением.

При заготовке сенажа в рулонах, упакованного в пленку, получается высококачественный корм, использование которого оправдано как 